\title{
Cloud Based SCADA System for Integrated Water Resources Development and Management Approach with Special Reference to Kannur District
}

\author{
V. Sampathkumar ${ }^{1 *}$ and P. Sridharan ${ }^{2}$ \\ ${ }^{1}$ Applied Electronics and Instrumentation Engineering, Vimal Jyothi Engineering College, Chemperi, Kannur-670632, \\ Kerala, India \\ ${ }^{2}$ Mechanical Engineering, Vimal Jyothi Engineering College, Chemperi, Kannur-670632, Kerala, India
}

*Corresponding author: vsampath@vjec.ac.in

\begin{abstract}
Canal irrigation is widely used source of water for irrigation. So, management of irrigation canal water is crucial factor in overall irrigation development. The conventional method is used to deliver water as per demand by the water users (farmers) in form of rotations. The conventional system involves lot of weaknesses, including forecast and actual flow or discharge. Errors may introduce in flow measurements and water content in reservoirs, also imbalances due to human and natural intervention are not considered in the conventional system. Due to this, users at the tail end endure more or shortage water. To provide efficient delivery and avert imbalance, canal automation plays crucial role in irrigation. This paper proposes remote flow control system based on Supervisory Control And Data Acquisition (SCADA). System monitors and controls required flow continuously, also monitors power status, upstream level, downstream level and system status. It initiates push communication in case of any uncertainty. Desired flow command given by remote SCADA automatically or by operator at main SCADA.
\end{abstract}

Keywords: IWRDM, Cloud computing, SCADA, RTU, Intelligent control

Agriculture is the mainstay of the Indian economy; almost three quarters of the country's working population are engaged in agriculture and about half of the gross national product is generated by agricultural production. Agricultural growth largely depends on water, which is the prime input. Rainfall is not quite dependable or helpful to agricultural development in India. Fourth generation: "Internet of things" With the commercial availability of cloud computing, SCADA systems have increasingly adopted Internet of things technology to significantly reduce infrastructure costs and increase ease of maintenance and 
integration. As a result, SCADA systems can now report state in near real-time and use the horizontal scale available in cloud environments to implement more complex control algorithms than are practically feasible to implement on traditional programmable logic controllers. This decentralization of data also requires a different approach to SCADA than traditional PLC based programs. When a SCADA system is used locally, the preferred methodology involves binding the graphics on the user interface to the data stored in specific PLC memory addresses. However, when the data comes from a disparate mix of sensors, controllers and databases the typical 1 to 1 mapping becomes problematic. A solution to this is data modeling as shown in Fig. 1. A concept derived from object oriented programming.

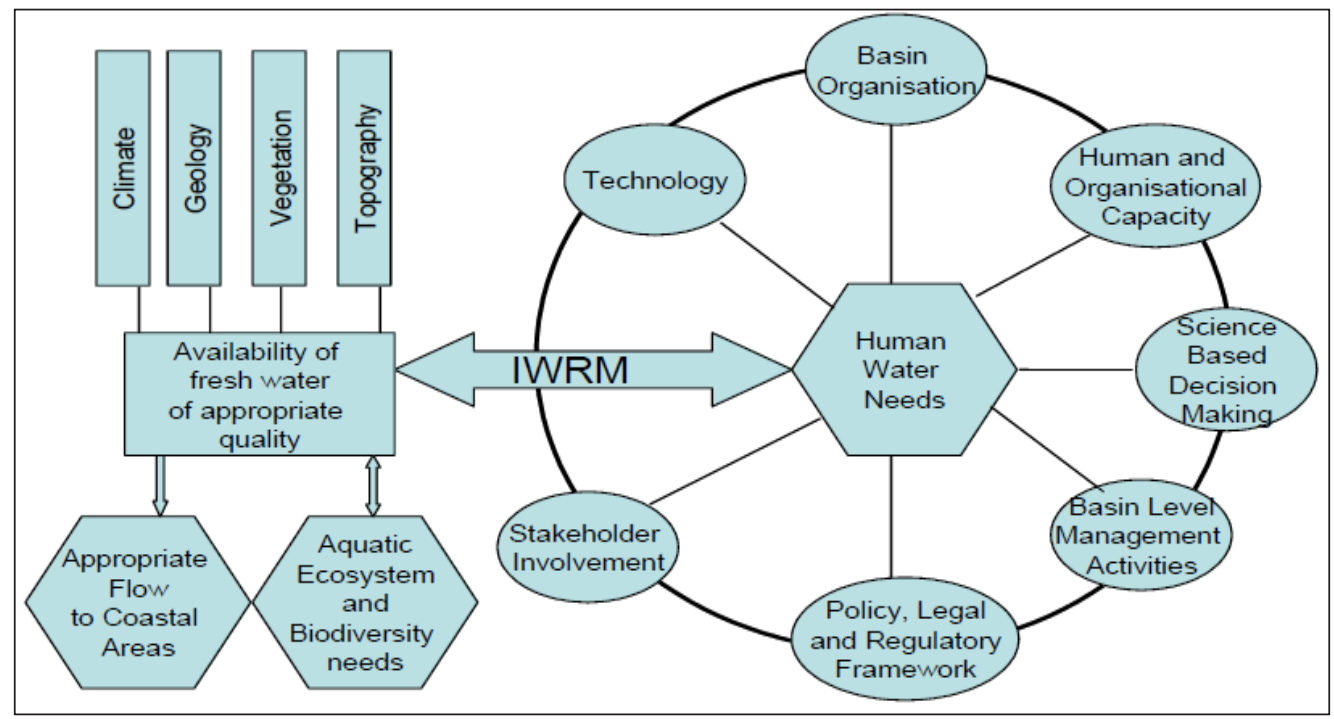

Fig. 1: Components of IWRA

In a data model, a virtual representation of each device is constructed in the SCADA software. These virtual representations ("models") can contain not just the address mapping of the device represented, but also any other pertinent information (web based info, database entries, media files, etc.) that may be used by other facets of the SCADA/IoT implementation. As the increased complexity of the Internet of things renders traditional SCADA increasingly "house-bound," and as communication protocols evolve to favor platform-independent, service-oriented architecture (such as OPC UA), it is likely that more SCADA software developers will implement some form of data modeling.

Kannur (Cannanore) district is a maritime district of northern Kerala with 23,312 ha. area under irrigation. Where the water is drained by rivers like the Valapattanam, Anjarakandy, Kuppam, Mahi, Thalasserry etc. The major irrigation scheme here is Pazhassi project with a fixed command area was 11525 ha of land where only 8125 ha of land has been benefited as on date. The monthly Potential Evapo Transpiration (PET) ranges from 124.5 to $170.6 \mathrm{~mm}$. which are lower than the monthly rainfall during the months of May to October indicating water surplus for possible recharge into groundwater regime during these months. Thalasserry and Kuthuparambu blocks having a stage of ground water development of 80.39 $\%$ and $77.24 \%$ respectively are demarcated as vulnerable area on consideration of depletion of ground water level according to The Kannur Water Conservation Society (KWCS). Since Dendritic being the common drainage pattern available, the water conservation activities can be planned with constructing 
rainwater harvesting, constructing check-dams and pits to lower the surface run-off of rainwater during monsoon. But the delivery of water according to the demand plays the key role. Which is achieved by the cloud based Supervisory control and data acquisition (SCADA) systems.

As a result of that it can state in real-time function and capable of implementing more complex control algorithms using the cloud environments along with PLC's. Further, the use of open network protocols such as Transport Layer Security (TLS) inherent in the Internet of Things technology, provides a more readily comprehensible and manageable security boundary than the heterogeneous mix of proprietary network protocols typical of many decentralized SCADA implementations. So this technology can pave way for the innovative approach Cloud based SCADA systems for irrigation in Kannur District of Kerala through the implementation of real time controls (RTC).

KERALA-"God's own country", the water rich State which has an average annual precipitation of around $3000 \mathrm{~mm}$, is facing water scarcity, falling ground water levels, drought, rivers running dry, shrinking wet lands and frustrated farmers. The people forget this water shortage as problem of floods creeps by then. It is the result of missed the bountiful monsoon water investment and mismanagement of this precious resource. About 52 billion cubic meters $(\mathrm{bcm})$ going waste to the sea during the monsoons against the estimated $60 \mathrm{bcm}$ average annual stream flow in the State. Obviously, very few major and medium storages are being constructed to detain the monsoon flows. The environmental groups and others have been vociferous against massive structures and needed only small structures to harness the monsoon flows. Hence a combination of major, medium and minor storages has to be further constructed in the State to utilize the available monsoon flows.

In this, both the concerns of supply management and demand management will be addressed. The decision makers, water professionals, environmental activists and the user groups in general all will be made aware of roles played by them to ensure the upkeep of quality and optimal utilization of the water. For the IWRDM to function effectively we need to move towards a cloud based Supervisory control and data acquisition (SCADA) system which is the fourth generation SCADA system. SCADA being a modern control system method here uses computers, networked data communications and Human Machine (HMI) user interfaces for high-level process supervisory management. We use peripheral devices such as Programmable Logic Controllers (PLC) and discrete PID controllers for the real-time control logic or controller control actions like the check dam shutter or valve opening (actuators). Which are performed by networked modules based on the demand from the farmers or based on the set points compared with the various sensors like level,

\section{LITERATURE SURVEY}

Kiran S. Shingote, Prof. Priti Shahane have proposed Modern growth in electronics systems, communication system, and information technology assist in designing canal automation system. The Canal irrigation is tremendously use source of water in irrigation. Microcontroller based system is very flexible for any modification required at site, while this system is cheaper than PLC can interface different modules easily. System involves intelligent Microcontroller based Remote Terminal Unit (RTU) which can communicate different sensors, communication modems, memory, ADC and different modules. In this paper we propose a microcontroller based design for flow control system for gate in canal automation. Flow control system consists of sub systems: RTU, Solar Power system, level measurement system, flow measurement system, gate actuator system, and communication system. In this paper more focus on flow 
control activities of Distributary, laterals and Direct Pipe Outlets (DPOs). Remote Terminal Unit monitors upstream level, downstream level, downstream flow, power status, gate opening, gate health and security. Geethu Ann Joseph, Elba Helen George has discussed the Canal irrigation system is the tool for monitoring and optimizing water flow in irrigation project. Managing canal irrigation system to achieve efficiency, equity and sustainability is a difficult task. Irrigation water is an input for agricultural production. The objective of this study is to analyze an automation system in an irrigation system to control the water distribution throughout the area. Efficiency can be improved by scientific water management techniques. The irrigation system is to be studied based on different automation techniques. By management of irrigation system predictable and equitable water is to be distributed to beneficiaries in time.

Shubham Sharma, Golu Yadav, Jaydeep Prajapati, Nitin Namdev, Vishal Sharma have discussed the Present scenario, availability of drinking water is very huge problem in many states including Madhya Pradesh. Many people have been suffering a lot to bring water in time. The monitoring of water can prevent the occurrence of stealing and leaking of water effectively for the household. In order to overcome the difficulty in the conventional system, the software based automated system using embedded controller is used for distributing the water to the people equally according to their utilization. The Embedded controller is already pre-programmed to do the operations by software and results are shown in our software.

Nowadays, the management of water is of paramount importance for modern societies due to the high water-availability requirements. The application of water management schemes requires the installation of water level data-acquisition systems in multiple, geographically isolated large-scale storage tanks of water distribution networks. Existing techniques for liquid level sensing have either been applied over a relatively small measurement range, or require special scientific equipment of high cost, or they are not convenient for transportation, installation and long-term maintenance in multiple large-scale water storage tanks of water distribution networks in cities, communities, etc.

In this paper, a review of prior art on liquid level sensing is initially presented. Then, the operational characteristics and performance of a novel capacitive-type water level measurement system are investigated through simulations and experimental tests conducted in two water storage tanks of a city-scale water distribution network. Here in our project ultrasonic level sensor is been used because of the flexibility and maintenance. Since it's a huge storing area ultrasonic level sensor is used. Ultrasonic level sensors are also part of automated flow control system. Level sensing system measures upstream level and downstream level of the gate. Both sensors are ultrasonic level sensors. Sensor transmits ultrasonic waves towards water and then measures time difference between transmitted of reflected waves. Reflected echoes are received by the receiver and converted into analog signals.

\section{PROPOSED RESEARCH AND METHODOLOGY}

The Proposed system is shown in Fig. 2.

\section{CLOUD STORAGE}

The cloud is the concept of using large arrays of remote Internet-based servers to store and handle your information. Your company's data is saved off-site, on the servers of a third-party hosted cloud service; this information can be accessed by connecting to the cloud server through your Internet connection. 
Although the cloud servers don't belong to your company, they are used as if they did. Think about it like renting a storage unit for items you want to keep around but can't fit in your garage. The space is yours to use as you wish, but you don't own it, and it's not located right next to your house. Now apply that metaphor to server hardware, IT support, maintenance costs, and software. While many cloud services are specifically meant as storehouses for data, some cloud-based SCADA systems are offered as a "service" - which is referred to as SaaS (Software as a Service). Instead of having the SCADA system software installed on local computers, the entire system and its data is stored and maintained in the cloud. SaaS companies offer their customers the power of software applications, off-site IT support and scalable server space all through the cloud.

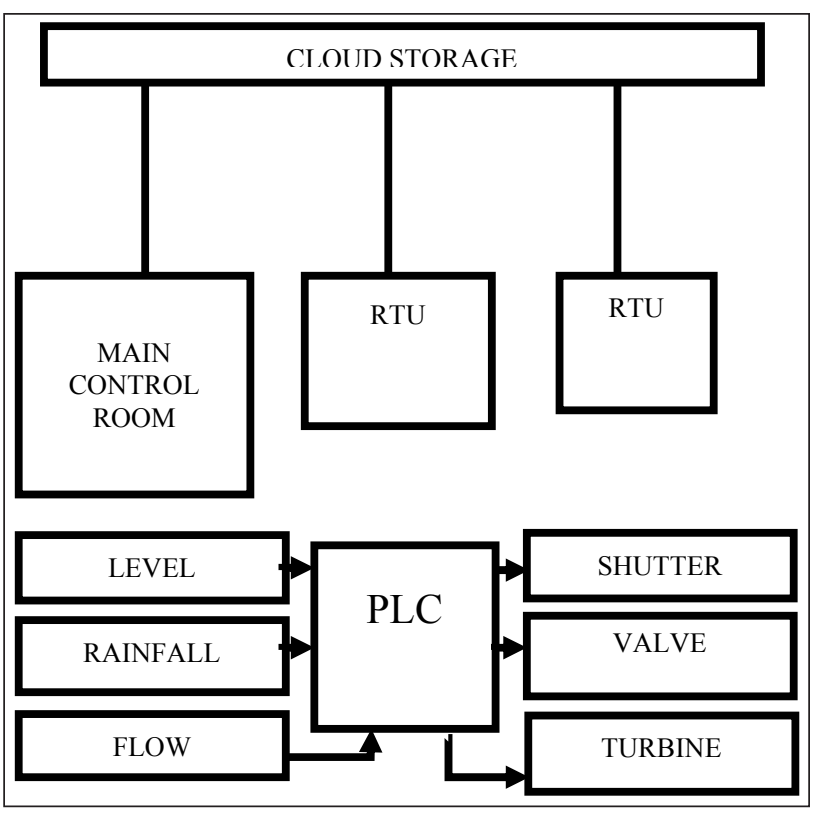

Fig. 2: Block diagram

The Benefits of Moving to the Cloud While most direct process SCADA system applications are remaining in-house, many sections of manufacturing plants are relocating to the cloud because the benefits outweigh the risks involved as shown in Fig. 3. Following are some of the benefits of the cloud that are encouraging companies to move Better Collaboration Since information in the cloud is easily accessible, multiple individuals at different levels of the company can collaborate on projects more easily ${ }^{[6]}$. This enables all parties to work together more efficiently, which increases the company's competitiveness. Ease of Upgrading and Adding Additional Applications Upgrading and installing new applications are extremely easy in the cloud. Once applications are upgraded or installed, they are instantly available to everyone with access

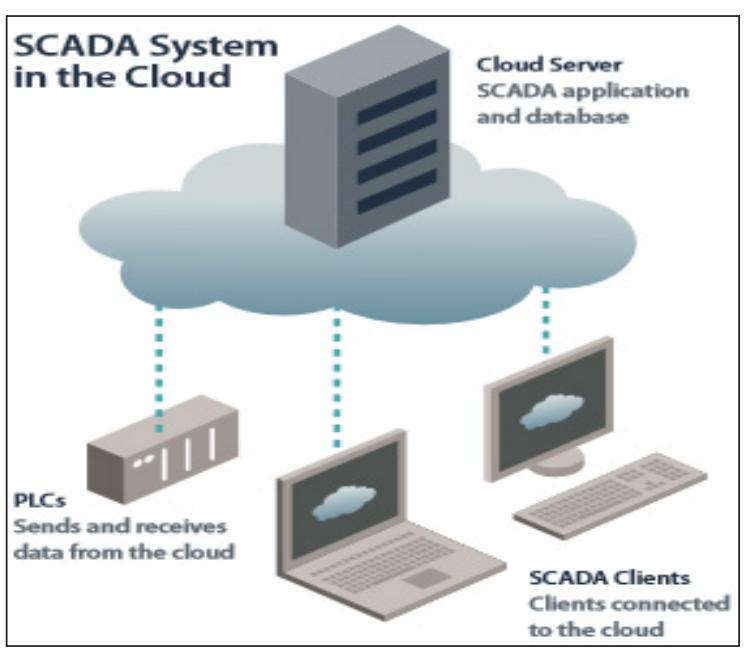

Fig. 3: Cloud Based SCADA to the network.

\section{RTU-Remote Terminal Unit}

We take a Remote Terminal Unit (RTU) which controls a modified stream. The location for this modified stream is selected using GRASS GIS, where GRASS (Geographic Resources Analysis Support System), is an open source software Geographic Information System (GIS) mainly used for geospatial data 
management and analysis, spatial modeling, image processing, visualization etc. The modified stream here means 10 to 20 meters of wide stream is enlarged to pond of 100-150 meters diameter with an inlet and outlet remaining as 20 meters. Similarly the width is also increased from 10 feet to 20 to 40 feet owing to more storage of water. We need to construct a check dam for the main stream flow which is in-turn controlled by the shutter. Here the water distribution to distant areas is done thought cement pipes by using gravitational forces. The end of the pipes are fitted with small hydro electric generators for power generation. Like this we have number of RTU's and in our project.

Almost all activities are under the control of RTU ${ }^{[1]}$. These activities are algorithm of flow control, power monitoring, actuator control, level monitoring, communication activities and security issues. PI (Proportional Integral) Algorithm, sdi-12 interface, GPS interface are easer designed with microcontroller programming than plc. RTU decodes flow command sent by main SCADA. Every RTU has its unique identification (id). If id Matched, RTU checks system health and mode of operation. RTU acquires level and flow continuously and do the correction in flow in predefined interval. The interval is depending on power availability, power status and application. Optimum interval can be set. Feedback control system is shown in Fig. 4. At every predefined interval RTU insures about expected flow captured by ultrasonic Doppler sensor. If there is any deviation between current flow and expected flow, it calculates and sends new gate opening using PI Controller algorithm. Then communicates with actuator controller and sends command to correct the gate opening. RTU is capable to monitor and control any disturbance due to upstream level change. Generally gate have wide deployment and in large quantity ${ }^{[2-3]}$. RTU have facility to synchronize local Real Time Clock (RTC) with Global Positioning System (GPS). RTU set RTC in a predefined interval. RTU is capable of storing all the parameters in nonvolatile flash memory as well as in micro Secure Digital (SD) card for 1 year in comma separated variables (CSV) format. This stored data can be easily retrieved and can be used for analysis propose $\mathrm{e}^{[4]}$.

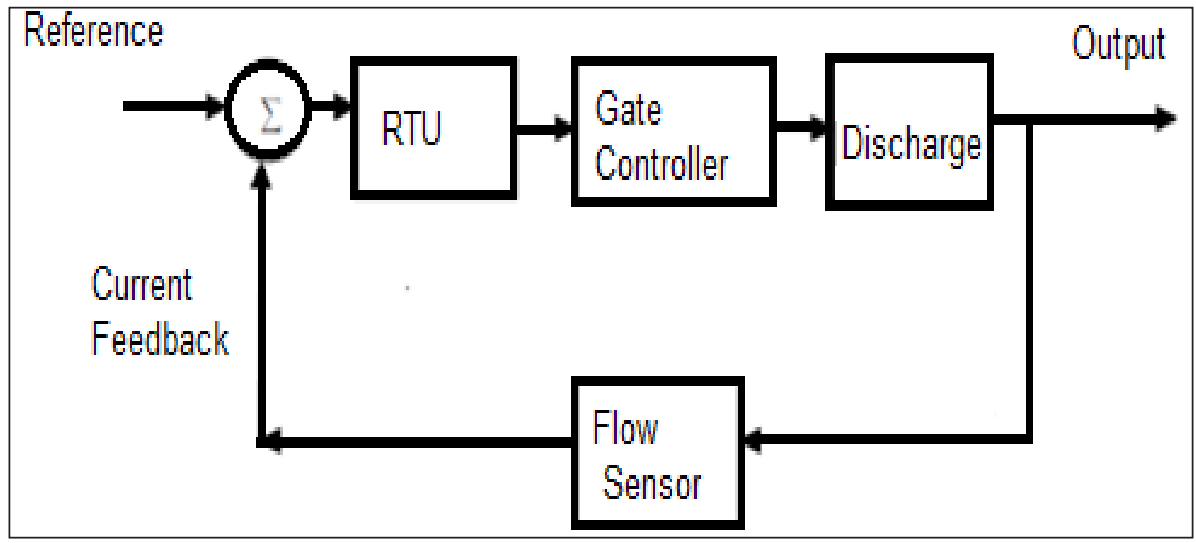

Fig. 4: Feedback Control System for Flow Measurement

\section{Main Control Room}

An operations center staffed by personnel charged with the responsibility for remotely monitoring and controlling a pipeline facility. CRM inspections are based on Control Rooms. A Control Room may be:

Secure Room at Company 
$\square$ Headquarters

$\square$ Open Office Cubicle

$\square$ Manager's Office

$\square$ Compressor/Pump Station Control

$\square$ Building

$\square$ Cab of Pick-Up Truck

$\square$ Kitchen in a Private Residence, etc.

This section applies to each operator of a pipeline facility with a controller working in a control room who monitors and controls all or part of a pipeline facility through a SCADA system [4-6]. Each operator must have and follow written control room management procedures that implement the requirements of this section Roles and responsibilities. Each operator must define the roles and responsibilities of a controller during normal, abnormal, and emergency operating conditions. To provide for a controller's prompt and appropriate response to operating conditions, an operator must define each of the following:

1. A controller's authority and responsibility to make decisions and take actions during normal operations;

2. A controller's role when an abnormal operating condition is detected, even if the controller

3. Is not the first to detect the condition, including the controller's responsibility to take specific actions and to communicate with others;

4. A controller's role during an emergency, even if the controller is not the first to detect the

5. emergency, including the controller's responsibility to take specific actions and to communicate with others; and

6. A method of recording controller shift-changes and any hand-over of responsibility between controllers.

\section{PLC}

In this work FX3U-128MR-ESManufactured by Mitsubishi. With the use of 3 input port and 3 output port A PLC is a solid state device designed to perform the logic functions previously accomplished by components such as electromechanical relays, drum switches, mechanical timers/counters etc. for the control and operation of manufacturing process equipment and machinery. Even though the electromechanical relay (control relays, pneumatic timer relays, etc.) have served well for many generations often under adverse conditions, the ever Increasing Sophistication and complexity of modern processing equipment require faster acting, more reliable control functions that electromechanical relays or timing devices cannot offer. The requirement of highly specialized, high-speed manufacturing processes created a demand for smaller, faster acting, more reliable, low power consuming, expandable, eliminating much of the hard wiring control devices called PLC's. Richard E. Morley, who was the founder of the Modicon Corporation, invented the first PLC in $1969^{[11]}$.

The PLC is also designed to operate in the industrial environment with wide ranges of ambient temperature, vibration, and humidity and is not usually affected by the electrical noise that is inherent in most industrial locations. Troubleshooting is simplified in most PLCs because they include fault indicators, blown- 
fuse indicators, input and output status indicators, and written fault information that can be displayed on the programmer. PC is often used for programming and monitoring the PLC, however, some PLC manufacturers also offer hand-held programming channels \& dedicated programmers ${ }^{[12]}$.

\section{Flow Measurement System}

Following are the different flow measurement techniques for an open channel.

1. Velocity-Area Method

2. Slope-Area Method

3. Stage-Discharge Method

As water through gates charged to farmers, flow computing at all stages needs to be more accurate. Among these techniques, Velocity-Area method is very accurate which uses stream velocity in $\mathrm{m} / \mathrm{s}$ and wetted cross-section area. Flow measurement system is based on acoustic Doppler shift principle. This flow measurement technique is very accurate technique. Conventional method uses Stage-Discharge Method and Bernoulli equation to calculate flow. In Velocity-Area Method flow can be calculated as follows:

$$
\mathrm{Q}=\mathrm{V} \times \mathrm{A}
$$

Where, $Q=$ discharge or flow $\left(\mathrm{m}^{3} / \mathrm{s}\right), V=$ velocity of water $(\mathrm{m} / \mathrm{s}), A=$ wetted cross section area $\left(\mathrm{m}^{2}\right)$

Doppler transducers usually operate at $640 \mathrm{kHz}$ to $1 \mathrm{MHz}$ frequency sensor transmits ultrasonic waves; waves are reflected by tiny particles in water or bubbles. Reflected signal captured by sensor the with shift a in frequency. If water flows Towards sensor, frequency will increase and water flows away from sensor frequency will decrease. In acoustic Doppler principle velocity profile acquires by Doppler shift of every cell. After that mean velocity is be calculated. This sensor also measures depth using a pressure sensor to calculate wetted cross section area. Flow is calculated using equation (1). These sensors

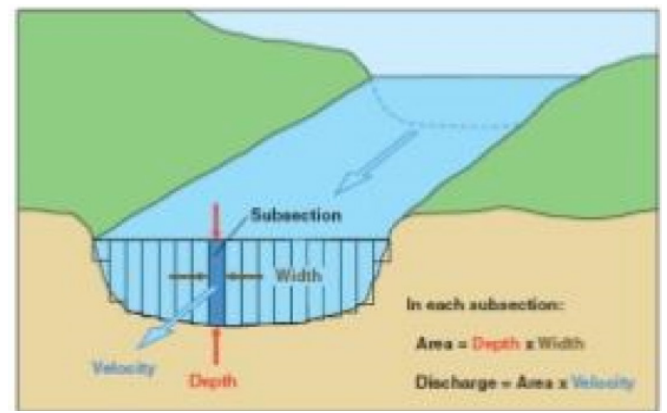

Fig. 5: Velocity-Area Method provide output an as SDI-12 (Serial Digital Interface with 1200 bits per second) which is specially designed protocol for hydrological and metrological instruments. Ultrasonic Doppler sensors can be used in partially full pipes or open channels with combined velocity and depth sensor as shown in Fig 5.

\section{Level Measurement System}

In our project, we use ultrasonic Sensor for Level Measurement. Level sensors are also part of an automated flow control system. Level sensing system measures upstream level and downstream level of the gate. Both sensors are ultrasonic level sensors. Sensor transmits ultrasonic waves towards the water and then measures the time difference between transmitted of reflected waves. Reflected echoes are received by the receiver and converted into analog signals. An ultrasonic wave speed is at the speed of sound and 
the time difference of transmitted and received pulses is proportional to the distance between the sensor and the surface to be measured as shown in equation (2).

$$
\mathrm{D}=(\mathrm{T} \times \mathrm{C}) \div 2
$$

Where, $\mathrm{D}=$ distance between sensor and wastewater,= time difference between transmitted and reflected wave $\mathrm{C}=$ speed of wave propagation $(\mathrm{m} / \mathrm{s})$

The transducer has a built-in temperature sensor which can provide temperature compensation for the measured values. With the known distance and reference level, actual level (L) is calculated.

$$
\mathrm{L}=\mathrm{E}-\mathrm{D}
$$

Where, $\mathrm{L}=$ water level $(\mathrm{m})$

$\mathrm{E}=$ distance from measuring device to zero level, $\mathrm{D}=$ distance from measuring device to water the surface.

The output of these sensors is 4-20 mA current or digital serial as shown in Fig. 6.

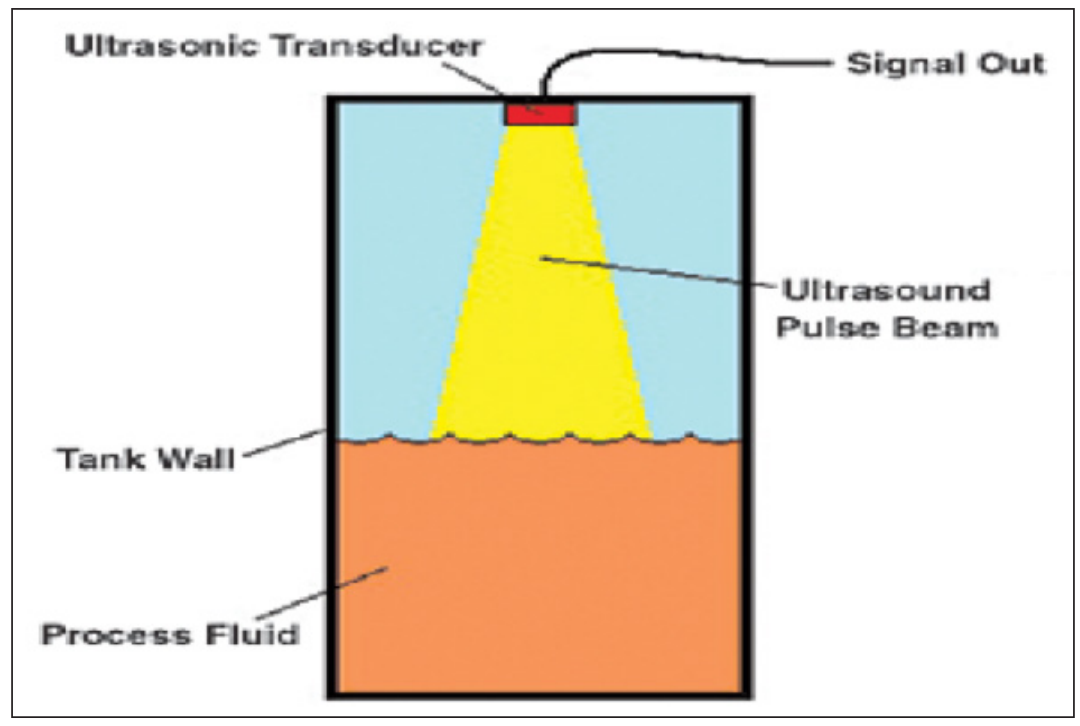

Fig. 6: Ultrasonic Sensor for Level Measurement

\section{Valve}

In our project, we use two solenoid valves two of them is used for irrigation purpose and another one to power generation. A solenoid valve is an electromechanically controlled valve. The valve features a solenoid, which is an electric coil with a movable ferromagnetic core in its Centre. This core is called the plunger. In the rest position, the plunger closes off a small orifice. An electric current through the coil creates a magnetic field. The magnetic field exerts a force on the plunger. As a result, the plunger is pulled toward the centre of the coil so that the orifice opens. This is the basic principle that is used to open and close solenoid valves. 
A solenoid valve is an electromechanical actuated valve to control the flow of liquids and gases. Solenoid valves are amongst the most used components in gas and liquid circuits. The number of applications is almost endless. Some examples of the use of solenoid valves include heating systems, compressed air technology, industrial automation, swimming pools, sprinkler systems, washing machines, dental equipment, car wash systems, and irrigation systems.

\section{Shutter}

The modified stream here means 10 to 20 meters of the wide stream is enlarged to a pond of $100-150$ meters diameter with an inlet and outlet remaining as 20 meters. Similarly, the width is also increased from 10 feet to 20 to 40 feet owing to more storage of water. We need to construct a check dam for the main stream flow which is in-turn controlled by the shutter. To raise the shutter we use dc motor. A DC motor is any of a class of rotary electrical machines that converts direct current electrical power into mechanical power. The most common types rely on the forces produced by magnetic fields. Nearly all types of DC motors have some internal mechanism, either electromechanical or electronic, to periodically change the direction of current flow in part of the motor( 1,7$)$.

A motor is an electrical machine which converts electrical energy into mechanical energy. The principle of working of a DC motor is that "whenever a current carrying conductor is placed in a magnetic field, it experiences a mechanical force". The direction of this force is given by Fleming's left-hand rule and its magnitude is given by $\mathrm{F}=\mathrm{BIL}$. Where, $\mathrm{B}=$ magnetic flux density, $\mathrm{I}=$ current and $\mathrm{L}=$ length of the conductor within the magnetic field.

Fleming's left hand rule: If we stretch the first finger, second finger and thumb of our left hand to be perpendicular to each other AND direction of magnetic field is represented by the first finger, direction of the current is represented by the second finger then the thumb represents the direction of the force experienced by the current carrying conductor.

\section{Turbine}

A water turbine is a rotary machine that converts kinetic energy and potential energy of water into mechanical work. Water turbines were developed in the $19^{\text {th }}$ century and were widely used for industrial power prior to electrical grids. Now they are mostly used for electric power generation water turbines are mostly found in dams to generate electric power from water kinetic energy.

Flowing water is directed on to the blades of a turbine runner, creating a force on the blades. Since the runner is spinning the force acts through a distance (force acting through a distance is the definition of work). In this way energy is transferred from the water flow to the turbine. Water turbine are divided in two groups reaction turbines and impulse turbines. The precise shape of water turbine blades is a function of the supply pressure of water and the type of impeller selected (10-12). Reaction turbines are acted on by water which changes pressure as it moves through the turbine and gives up its energy they must be encased to contain the water pressure (or suction) or they must be fully submerged in the water flow. Newton's third law describes the transfer of energy for reaction turbines. Most water turbines in use are reaction turbines and are used in low ( $<30 \mathrm{~m}$ or $100 \mathrm{ft}$ ) and medium (30-300 $\mathrm{m}$ or 100-1,000 ft) head applications. In reaction turbine pressure drop occurs in both fixed and moving blades. It is largely used in dam and large power plants, Impulse turbines change the velocity of a water jet. The jet pushes on 
the turbine's curved blades which changes the direction of the flow. The resulting change in momentum (impulse) causes a force on the turbine blades. Since the turbine is spinning, the force acts through a distance (work) and the diverted water flow is left with diminished energy. An impulse turbine is one which the pressure of the fluid flowing over the rotor blades is constant and all the work output is due to the change in kinetic energy of the fluid.

\section{RESULTS AND DISCUSSION}

In this new millennium, there are growing concerns and periodic warnings that we are moving into an era of water scarcity. With increasing demand for food and competing use within the water sector, the pressure is on irrigation professionals to manage water efficiently. So we have created a cloud-based SCADA System for Integrated water resources development and management in the Kannur district of Kerala and created an efficient method for irrigation inside the full district. In this method, we can have several RTU's that can enhance the efficient irrigation for several parts of the district.

We have done this project to have an efficient method to provide the water to several parts of the district in a schematic way. Kannur district has plenty of resources of water but at summer season people are suffering from water scarcity. This problem can be avoided by our project using some simple methods of water harvesting. The main rivers can be used for this project by creating several RTU's as mentioned above. We have created a prototype for this project by automating this system by using the cloud-based SCADA program and PLC's. We have obtained certain results based on our prototype that have been made and this prototype results in how to save and use water for different purposes in our district. We have used cloud-based SCADA for controlling several RTU's and this can be programmed by a SCADA program. The SCADA program can have its own icons that we can easily control with. The SCADA programs can be done by simple switches and simple programs as shown in Fig. 7.

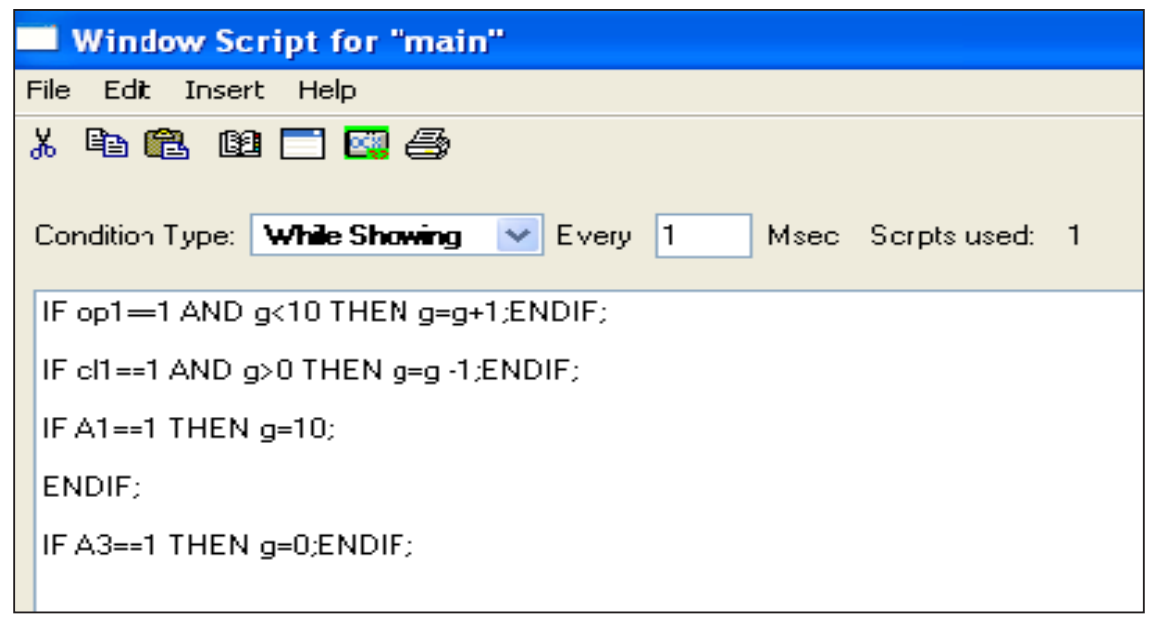

Fig. 7: SCADA program for the RTU for IWRDM

Above shown is the SCADA program that we are dealing with in this program we can have a switch that can enhance us for automation of the RTU or we can run the RTU in manual mode. We can also have the controlling buttons for opening and closing the gates that we are provided to the RTU and there is 
also a controller for the solenoid valve and two sensors for the high and low level of water as shown in Fig. 8. We can program this switches and sensors by a simple program.

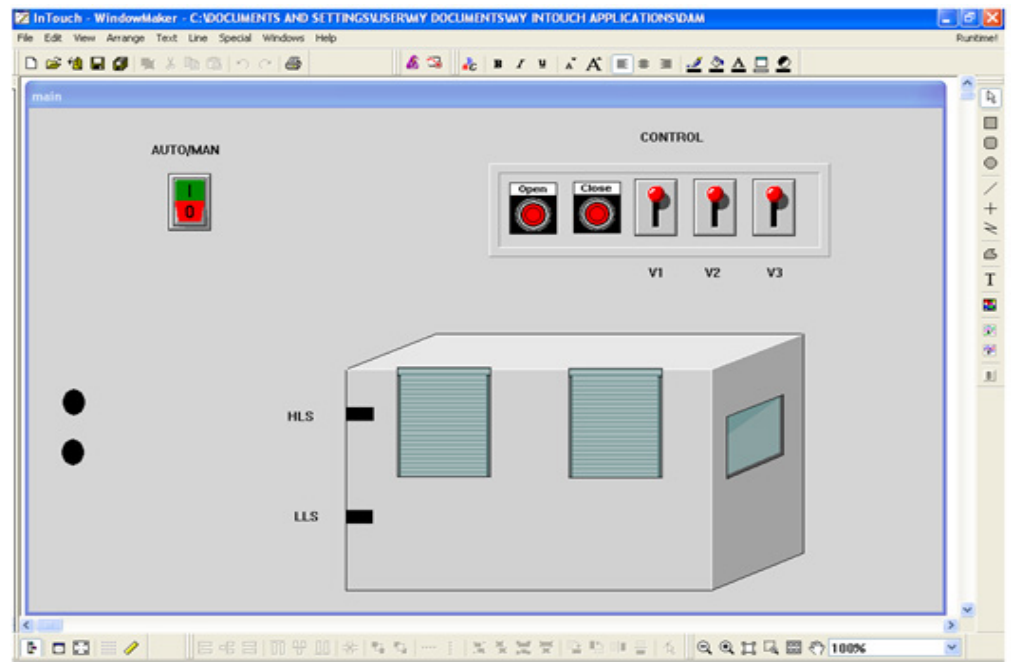

Fig. 8: SCADA program for IWRDM

The above shown program can perform the task of programming for the operation of the gate and several controllers that we have been used. This program can function the SCADA system that we have been used as shown in Fig. 9 and 10.

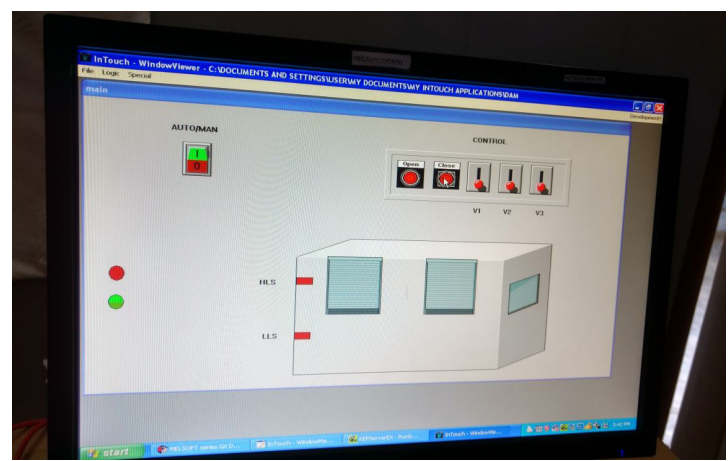

Fig. 9: SCADA system when the shutter is opening

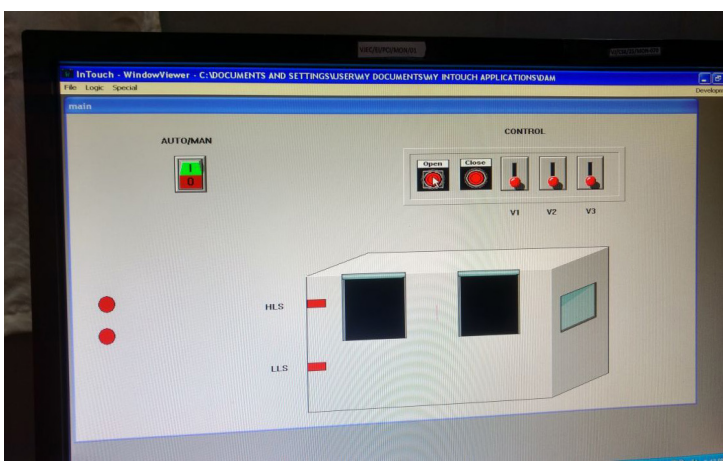

Fig.10: SCADA system when shutter closed

The SCADA is directly controlling the RTU with the help of PLC's. The RTU's are remote terminal units that can be made in the river at several areas for the water conservation and efficient use of water. The RTU can have the shutter for water inlet from one side and another for water outlet. The water is collected in a large area and we are provided with many valves that can provide water to irrigation purpose, energy production, etc... is made such the water is to be reserved and excess water is to be given out and this is to be the input of the next RTU. Certain RTU can be illustrated as Fig. 11. RTU with two shutters and each shutter have a motor and three solenoid valves and a level sensor. 
The PLC's are the certain instrument used to interface with the SCADA that we have used. The plc is associated with the RTU and it can be operated with simple ladder program to perform a RTU functions. We have used the plc of Mitsubishi for our project works. The PLC program is downloaded from the pc through a cable called RS232 as shown in Fig. 12 and there are memory allocated in the plc program that can be used in the SCADA program.

\section{CONCLUSION}

SCADA being a modern control system method here used with computers, networked data communications and Human Machine (HMI) user interfaces for high-level process supervisory management. We used peripheral devices such as Programmable Logic Controllers (PLC) and discrete PID controllers for the real-time control logic or controller control actions like the check dam shutter or valve opening(actuators). Which are performed by networked modules based on the demand from the farmers or based on the set points compared with the various sensors like level, temperature, rainfall, moisture content, water flow etc, This work would be an answer to drought, flooding, and salinity intrusion. We have done this project to encompass an efficient method to provide the water to several parts of the district in a systematic way. Kannur district has plenty of water resources within the proximity, but during the summer season, people are suffering from water scarcity. This problem can be avoided by our project using some simple methods of water harvesting.

Future enhancement of this work is that it can be widened for the entire state of Kerala, the Kerala State Electricity Board (KSEB) can be clubbed with the water distribution.

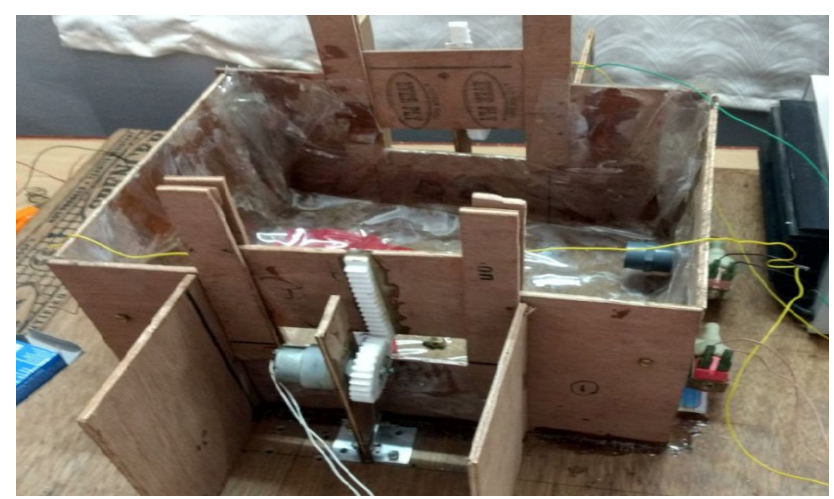

Fig. 11: RTU when shutter is opened

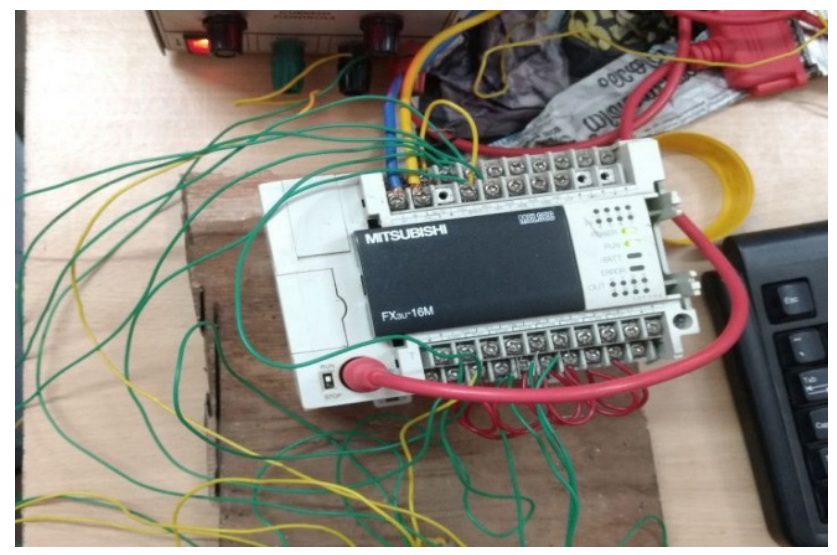

Fig. 12: PLC that have been used in the project

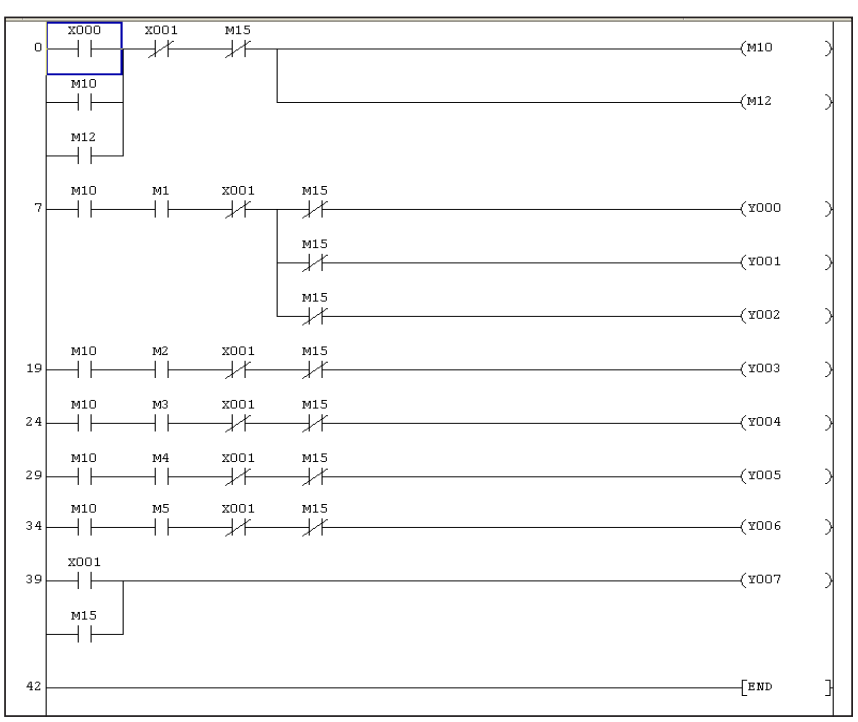

Fig. 13: Ladder program for the plc. 


\section{ACKNOWLEDGMENTS}

We sincerely acknowledge the financial support extended by Kerala State Council for Science, Technology \& Environment, Sasthra Bhavan, Pattom P O, Thiruvananthapuram 695004 (FILE O:147SPS60/2016KSCSTE) and the Management, Principal and faculty members from AEI department of Vimal Jyothi Engineering College, Jyothi Nagar, Chemperi,Kannur-670632, for completing this work in successful manner.

\section{REFERENCES}

1. Kiran S. Shingote, Priti Shahane, 2016. Microcontroller Based Flow Control System for Canal Gates in Irrigation Canal Automation, IEEE 6 ${ }^{\text {th }}$ International Conference on Advanced Computing, 2016.

2. Mahesh Nandania, 2013. "A Review Paper of Automatic Canal Gate Control of 3-Phase Induction Motor with PLC and VFD, Powered by Solar System and Monitoring by SCADA," International Journal of Emerging Trends in Electrical and Electronic, 1(1).

3. Anil B. Mandavia, "Modernization of irrigation system operational management by way of canal automation in India," Chief Engineer, Management Information System Sardar Saroar Narmada Nigam Limited, Gujrath, India.

4. Abu-El Magad, M., Eman Kamel and Khaled Kamel, 1997. "Economic Assessment of an Irrigation Canal Automation and Control Project," International Symposium on Intelligent Control, 16-18 July 1997, Istambul, Turkey.

5. Bautista, E., ASCE, A.M., Clemmens, A.J., ASCE, A.M. and Strand, R.J. 2006. "Salt River Project Canal Automation Pilot Project: Simulation Tests," Journal of Irrigation and Drainage Engineering, March/ April 2006.

6. Zhiliang Ding, Changde Wang, Guangming Tan and Guanghua Guan, 2009. "The Application of the Fuzzy Self-Adaptive PID Controller to the Automatic Operation Control of Water Transfer Canal System," Second International Conference on Intelligent Computation Technology and Automation, 2009.

7. Rahul N Deshmukh and Dr. M.P. Deshmukh, 2014. "Canal Automation System using Crouzet PLC," SSBT's College of Engineering and Technology Bombhori, Jalgaon, International Journal of Advanced Information Science and Technology, 24(24).

8. Manuel Rijo, 2003. "Local Automatic Control Modes in an Experimental Irrigation Canal," Irrigation and Drainage Systems, 17: 179-193.

9. Noha Samir Donia, 2002. "Development of El-Salam Canal Automation System," Environmental Studies and Researches Institute, Ain Shams University, Cairo, Egypt. Journal of Water Resource and Protection, 4: 597-604.

10. Geethu Ann Joseph, Elba Helen George, 2016. Effective Water Distribution by MIS and Canal Automation, International Journal of Scientific and Engineering Research, 4(3):79.

11. Shubham Sharma, Golu Yadav, Jaydeep Prajapati, Nitin Namdev, Vishal Sharma, 2016. Design of Automatic Domestic Service Water Supply System And Its Implementation, International Education and Research Journal, 2(9). 